\title{
Corrigendum
}

Danijela Serbic*, Jun Zhao and Jiafan He

\section{Corrigendum to: The role of pain, disability and perceived social support in psychological and academic functioning of university students with pain: an observational study}

https://doi.org/10.1515/ijamh-2021-2141

Corrigendum to: Danijela Serbic, Jun Zhao and Jiafan He. The role of pain, disability and perceived social support in psychological and academic functioning of university students with pain: an observational study. International
Journal of Adolescent Medicine and Health, 2020. (DOI: https://doi.org/10.1515/ijamh-2019-0032)

The wrong Table 2 was submitted during the revision by mistake. Correct Table 2 is presented below.

Authors and Publisher apologize for any inconvenience caused.

Table 2: Group comparisons statistics.

$\begin{array}{rrrrr}\text { Chronic pain } & \text { Acute pain } & \text { No pain } & \text { Inferential } & \text { Effect size } \\ \mathrm{n}=96 & \mathrm{n}=102 & \mathrm{n}=113 & \text { statistics/ } & \eta^{2} \\ \text { M, SD } & \text { M, SD } & \text { M, SD } & \text { p-Value } & \end{array}$

\begin{tabular}{|c|c|c|c|c|c|}
\hline \multicolumn{6}{|l|}{ ANOVA main effects: } \\
\hline Anxiety & $7.63(3.56)$ & $6.69(3.25)$ & $5.63(3.06)$ & $9.67^{\star \star \star}$ & 0.059 \\
\hline Depression & $5.41(3.87)$ & $4.36(3.15)$ & $4.19(2.72)$ & $4.14^{\star}$ & 0.026 \\
\hline PSS_Overall & $4.95(1.12)$ & $5.24(1.04)$ & $5.39(1.11)$ & $4.23^{\star}$ & 0.027 \\
\hline PSS_Family & $4.83(1.30)$ & $5.12(1.24)$ & $5.41(1.24)$ & $5.53^{\star \star}$ & 0.035 \\
\hline PSS_Friends & $5.05(1.21)$ & $5.36(1.18)$ & $5.45(1.16)$ & $3.31^{\star}$ & 0.021 \\
\hline PSS_Significant other & $4.99(1.28)$ & $5.23(1.15)$ & $5.32(1.15)$ & 2.11 & 0.014 \\
\hline Chronic vs. Acute group: & & & & $\mathbf{t}$ & $d$ \\
\hline Pain intensity (past week) & $2.79(2.38)$ & $1.89(1.79)$ & & $-2.99 * \star$ & 0.43 \\
\hline Pain frequency (3 months) & $3.95(2.36)$ & $2.53(1.81)$ & & $-4.73^{\star \star \star}$ & 0.68 \\
\hline Pain intensity (3 months) & $3.59(2.16)$ & $2.75(2.10)$ & & $-2.77^{\star \star}$ & 0.39 \\
\hline Disability & $12.45(11.51)$ & $11.10(10.75)$ & & -0.86 & 0.12 \\
\hline \multirow[t]{2}{*}{ Academic $^{a}$} & $7.34(7.57)$ & $5.61(5.97)$ & & -1.79 & 0.25 \\
\hline & & & & p-Value ${ }^{b}$ & $95 \% \mathrm{Cl}$ \\
\hline Anxiety & $7.63(3.56)$ & $6.69(3.25)$ & & 0.135 & $(-0.18,2.06)$ \\
\hline Depression & $5.41(3.87)$ & $4.36(3.15)$ & & 0.098 & $(-0.14,2.23)$ \\
\hline
\end{tabular}

ANOVA, analysis of variance; PSS, perceived social support; $M$, mean; SD, standard deviation; $F, F$ distribution statistic; $\eta^{2}$, eta squared; $\mathrm{Cl}$, confidence intervals; $\mathrm{t}$, $\mathrm{t}$ test; $\mathrm{p}$, calculated probability; $d$, Cohen's $d .{ }^{\star} \mathrm{p}<0.05,{ }^{\star \star} \mathrm{p}<0.01,{ }^{\star \star \star} \mathrm{p}<0.00 .{ }^{\mathrm{a}}$ Interference with academic functioning. ${ }^{b}$ Anxiety and depression comparisons are from ANOVA post hoc test (Bonferoni and Games-Howell respectively).

*Corresponding author: Danijela Serbic, Department of Psychology, Royal Holloway University of London, Egham, TW20 0EX, Surrey, UK, E-mail: danijela.serbic@rhul.ac.uk Jun Zhao and Jiafan He, Department of Psychology, Royal Holloway University of London, Egham, Surrey, UK 